\title{
Prácticas parentales como predictoras de la ideación suicida en
}

adolescentes colombianos

\section{(1)Psicogente ISSN 0124-0137 EISNN 2027-212X}

Artículo de Investigación Copyright 2018 by Psicogente

\section{Correspondencia de autores:}

mbahamon@unisimonboliva.edu.co yalarcon1@unisimonbolivar.eu.co lireyes@unisimonbolivar.edu.co atrejos@uninorte.edu.co iuribe@ucol.mx carolina.garcia@unad.edu.co

Recibido: $20-05-16$

Aceptado: 26-05-17

Publicado: 01-01-18

\section{Parenting practices, predictor of Colombian adolescents suicidal} ideation

\author{
Marly Johana Bahamón iD, Yolima Alarcón-Vásquez iD, Lizeth Reyes Ruiz3 iD \\ Universidad Simón Bolívar, Barranquilla, Colombia \\ Ana María Trejos Herrera \\ Universidad del Norte, Barranquilla, Colombia \\ J. Isaac Uribe Alvarado \\ Universidad de Colima, Estado de Colima, México \\ Carolina García Galindo \\ Universidad Nacional Abierta y a Distancia, Bogotá, Colombia
}

Resumen

Objetivo: El propósito del presente estudio fue determinar en qué medida las prácticas parentales paternas y maternas predicen la ideación suicida en un grupo de hombres y mujeres adolescentes colombianos

Método: Participaron 328 estudiantes adolescentes inscritos en escuelas públicas. Se utilizó la escala de Prácticas Parentales para Adolescentes (PP-A) elaborada por Andrade y Betancourt y una escala para medir la Ideación Suicida, constituida por ocho ítems que refieren a ideas de quitarse la vida

Resultados: Indican la existencia de correlaciones entre ideación suicida y control psicológico materno $\left(0,183^{* *}\right)$ e imposición $(-$ , $\left.282^{* *}\right)$ en los hombres y entre ideación suicida y autonomía $\left(-, 383^{* *}\right)$, control psicológico materno $\left(, 302^{* *}\right)$ e imposición $\left(, 383^{* *}\right)$ en mujeres.

También se evidenció que las dimensiones que predicen en mayor medida la ideación suicida en hombres y mujeres son el control psicológico materno y la imposición paterna.

Conclusiones: Se discute el papel de algunas prácticas parentales que pueden constituirse como posible factor de riesgo para disponer a los jóvenes hacia el suicidio

Palabras Claves:

Prácticas parentales, Ideación suicida, Control parental, Adolescencia

\section{Abstract}

Objective: This study aims to determine how parental and maternal parenting practices may predict suicidal ideation in a group of Colombian male and female teenagers.

Method: 28 teenagers who belong to public schools were sampled. 7-items related to wanting to take one's own life, (PP-A) Parenting Practices scale for teenagers was used.

Results: Show correlations between suicidal ideation and maternal psychological control $\left(0,183^{* *}\right)$ and impose $(-, 282 * *)$, in men between suicidal ideation and autonomy $\left(-, 383^{* *}\right)$, in women, maternal psychological control (, 302**) and impose (, 383**). Also, it is shown that maternal psychological control and parental impose are evident aspects which predict further suicidal ideation in men and women.

Conclusion: The role of some parenting practices can become a suicidal risk factor for young people.

Key words: Parenting practices, Suicidal ideation, Parental control, Adolescence

Como citar este artículo (APA):

Bahamón, M., Alarcón-Vásquez, Y., Trejos, A.M., Reyes, L., Uribe, J. \& García, C. (2018). Prácticas parentales como predictoras de la ideación suicida en adolescentes colombianos. Psicogente, 21(39), 50-61. http://doi.org/10.17081/psico.21.39.2821 


\section{Marly Johana Bahamón, Yolima Alarcón-Vásquez, Ana María Trejos Herrera, Lizeth Reyes Ruiz, J. Isaac Uribe Alvarado, Carolina García Galindo}

\section{INTRODUCCIÓN}

El suicidio se ha constituido en las últimas décadas como un problema de salud que afecta a un gran número de personas, además del contexto próximo en el que se da el hecho. Este es considerado como una de las principales causas de morbi-mortalidad a nivel mundial (Caycedo, Arenas, Benítez, Cavanzo, Leal \& Rossini, 2010). De manera particular en América Latina, los países con mayores tasas son Cuba, Brasil y Colombia. En este último, las cifras de suicidio empezaron a descender desde el año 2000 manteniendo dicha dinámica hasta el año 2006. No obstante, a partir del año 2007 se observó que la situación empezó a aumentar progresivamente, alertando nuevamente a la población. En Colombia, de acuerdo con un reciente reporte realizado por el Instituto de Medicina Legal y Ciencias Forenses (2015), la tasa de suicidio de Colombia fue de 5,22 por cada 100 mil habitantes mayores de cinco años, y 16 departamentos reportaron tasas superiores a la nacional.

A pesar de la representación compartida sobre la muerte como un fenómeno natural e inevitable para cualquier ser humano, que un individuo asuma esta decisión como única opción para detener su sufrimiento traslada fuertes interrogantes sobre las habilidades subjetivas de la persona para hacer frente a sus problemas, además, de la incapacidad del medio social para contener a quién requiere de una intervención y acompañamiento para hallar otras alternativas de solución (Casullo, Bonaldi \& Férnandez, 2000).

En este orden de ideas, el suicidio puede considerarse como un fenómeno complejo que influye de manera contundente en el entorno, el contexto y las futuras generaciones (Atay, Eren \& GündoĞar, 2012). Este hecho se relaciona frecuentemente con las etapas vitales del ser humano y uno de los grupos etáreos más afectados por este flagelo son los adolescentes, así como los adultos mayores.

Este estudio se concentró de manera específica en la adolescencia teniendo en cuenta que las cifras son más altas en esta etapa, además de las serias implicaciones en el contexto relacional debido a los significados atribuidos regularmente a la juventud, el impacto generado en familiares y pares que ponen en mayor riesgo al grupo de personas alrededor para que la conducta se imite.

La adolescencia como etapa de transición entre la infancia y la adultez, se caracteriza por múltiples transformaciones que allí se gestan. Desde la integración a un marco contextual y cultural, la afirmación de la identidad, la generación de conductas autónomas, la definición de la identidad sexual hasta la interacción con pares, entre otros, se constituyen en tareas que demandan al sujeto de mayor esfuerzo e implicación. La adolescencia se ha considerado como etapa de múltiples conflictos de construcción y deconstrucción en la cual el individuo se expone a la crisis vital tratando de vincularse al mundo como adulto aun teniendo características propias de la niñez, como la falta de autonomía y la necesidad de seguridad. En la adolescencia, los conflictos surgen con mayor arraigo y es la persona misma quien debe resolverlos, no obstante es fundamental considerar los recursos psicológicos y familiares con que cuenta para ello. 


\section{Marly Johana Bahamón, Yolima Alarcón-Vásquez, Ana María Trejos Herrera, Lizeth Reyes Ruiz, J. Isaac Uribe Alvarado, Carolina García Galindo}

En este orden de ideas, son múltiples los riesgos que se presentan en esta etapa, cuestiones como el consumo de alcohol y de sustancias psicoactivas, las conductas sexuales desprotegidas y el suicidio pueden considerarse como fenómenos relevantes. En este marco es importante considerar que el suicidio se ha convertido progresivamente en una problemática que afecta cada vez más a la población y de manera acentuada a las y los adolescentes.

De manera general, la conducta suicida consta de tres componentes: la ideación suicida, el intento suicida y el suicidio. La ideación suicida como primera manifestación de la conducta suicida se expresa en ideas o pensamientos de intencional auto-daño o muerte. Estudios de autopsia psicológica informan que la mayoría de suicidios se completan en el primer intento (Cavanagh, Carson \& Sharpe, 2003; Suominen, Isometsä, Suokas, Haukka, Achte, \& Lönnqvist, 2004), razón por la cual, es importante considerar la ideación suicida y su asociación con el suicidio consumado (Casey, Dunn \& Kelly, 2006). Por otro lado, el intento suicida es considerado como "el conjunto de conductas propias y voluntarias realizadas por el sujeto tendientes a la culminación de la vida, y el suicidio, es definido como la muerte que es resultado directo o indirecto de comportamientos ejecutados por la propia víctima la que es consciente de la meta a lograr" (Casullo et al., 2000, p.20).

La conducta suicida en el adolescente ha sido ampliamente estudiada por diferentes autores, identificando características que estos comparten y que son consideradas como factores riesgo, entre las cuales se destacan: exposición a situaciones familiares adversas, presencia de psicopatología, abuso de sustancias, baja autoestima, impulsividad, desesperanza, problemas afectivos y amorosos, problemas socioeconómicos, falta de comunicación y confianza con los padres, maltrato, antecedente de suicidio en la familia, baja tasa de supervisión de la madre, problemas de cohesión familiar, agresividad verbal e irritabilidad en el estado de ánimo de sus familiares, la vivencia de que sus familiares no comparten juntos su tiempo libre, no tener con quien contar en situaciones difíciles, sentimientos de soledad, tristeza, depresión, baja autoestima, poco soporte social y la existencia de problemas afectivos en general. Contrario a las características mencionadas se han identificado como factores protectores la relación con los padres, el apoyo y la atención familiar, específicamente de la madre (Cortés, Aguilar, Suárez, Rodríguez \& Salvador, 2011; Palacios \& Andrade, 2008; Rivera \& Andrade, 2006; Salvo \& Melipillán, 2008; Santos, Ferrera, González, Viñas \& Mauriset, 1997).

Sobre la ideación suicida como variable de estudio se evidencia que el recorrido es menor y los datos son menos prolíficos. No obstante, una de regularidades que se evidencia es la familia (ambiente y relaciones) como factor de riesgo o como factor protector. En este sentido, Cummings y Davies (2002) afirman que cuando un adolescente es expuesto a ambientes familiares incongruentes o disfuncionales es más probable que desarrolle desajustes emocionales o cognitivos. Así, por ejemplo, la carencia de redes de apoyo en el ambiente familiar en los adolescentes afecta su vinculación psicosocial, el pobre funcionamiento familiar, la presencia de crisis familiares, la disfuncionalidad, la poca expresividad, la mala comunicación y la desorganización familiar en lo que respecta a un consenso de normas pueden considerarse como factores de riesgo o predictores para la presencia de ideación suicida entre otros problemas (Álvarez, Ramírez, Silva, Coffin, \& Jiménez, 2009; Herrera \& Avilés, 2000; Pérez-Olmos, Ibáñez-Pinilla, Reyes-Figueroa, Atuesta-Fajardo \& 


\section{Marly Johana Bahamón, Yolima Alarcón-Vásquez, Ana María Trejos Herrera, Lizeth Reyes Ruiz, J. Isaac Uribe Alvarado, Carolina García Galindo}

Suárez-Díaz, 2008; Reyes \& Torres, 2001; Ferran, Canals, Gras \& Domènech-Llaberia, 2002; SánchezSosa, Villarreal-González, Musitu \& Martínez, 2010; Sook, Young \& Schepp, 2005; Toro, Paniagua, González \& Montoya, 2009; Suominen et al., 2004).

En el campo de la parentalidad, Andrade y Betancourt (2008) señalan que los abordajes teóricos se han concentrado en dos líneas: los estilos y las prácticas parentales. “...Los estilos parentales se refieren al clima emocional en el que los padres forman a sus hijos, las prácticas parentales aluden a las conductas específicas que utilizan los padres para educar a sus hijos" (Darling \& Steinberg, 1993, citado en Andrade \& Betancourt, 2008). Además señalan que las dimensiones estudiadas tanto en los estilos como en las prácticas parentales son el apoyo y el control.

De manera concreta, en el estudio sobre las prácticas parentales y su relación con la ideación suicida los reportes de trabajos investigativos son menores. En esta línea, Florenzano, Valdés, Cáceres, Santander, Aspillaga y Musalem (2011) realizaron una investigación con jóvenes chilenos entre los 15 y 19 años de edad para identificar factores de los estilos parentales asociados a la ideación suicida. Los resultados indicaron que aquellos adolescentes con padres que presentaban un alto nivel de hostilidad marital encubierta tenían mayor probabilidad de ideación suicida alta. Se registraron nueve factores protectores en común para los grupos con y sin ideación suicida: autoestima, aceptación parental, autonomía psicológica parental, calidad en la relación con la madre, calidad en la relación con el padre, amabilidad parental, expresión de afectos físicos, monitoreo paterno y participación en decisiones familiares. De acuerdo con lo anterior, los adolescentes cuyos padres ejercen autonomía psicológica, aceptación parental, expresión de afectos físicos, amabilidad parental y monitoreo paterno poseen menor ideación suicida que los adolescentes cuyos padres carecen de estas conductas.

En esta línea, Lai, Kwok y Shek (2008) estudiaron la relación entre la desesperanza, el funcionamiento familiar percibido y la ideación suicida en estudiantes chinos. Los resultados mostraron una relación positiva entre la desesperanza y la ideación suicida, en tanto, el funcionamiento familiar percibido mundial tuvo una relación negativa con la ideación suicida. Las diferentes dimensiones del funcionamiento familiar que se relacionaron diferencialmente con la ideación suicida fueron: el conflicto y la armonía, la preocupación de los padres y el control parental. Un fuerte predictor de la ideación suicida fue la preocupación de los padres y el control parental.

En otro estudio respecto a la ideación suicida y los estilos parentales, Pérez, Uribe, Vianchá, Bahamón, Verdugo y Ochoa (2013) reportan que de un grupo de 172 hombres y 226 mujeres adolescentes entre 15 y 17 años, la ideación suicida y los sentimientos de soledad se presentan en mayor porcentaje en las mujeres que en los varones, también informaron correlaciones significativas entre el control psicológico de la madre con la ideación suicida en mujeres y que, el control psicológico materno como la falta de afecto y comunicación paterno predicen en cierta medida la ideación suicida en hombres y mujeres adolescentes.

Finalmente, Sarmiento y Aguilar (2011) determinaron que los predictores de la ideación suicida fueron la autoestima y la frecuencia de conflictos con la madre, siendo el estilo negligente el que mostró la mayor correlación con la ideación suicida. 


\section{Marly Johana Bahamón, Yolima Alarcón-Vásquez, Ana María Trejos Herrera, Lizeth Reyes Ruiz, J. Isaac Uribe Alvarado, Carolina García Galindo}

Tomando como referencia los estudios sobre la influencia familiar en la ideación suicida de adolescentes y, considerando que las madres y padres de familia ejercen influencia en sus hijos e hijas adolescentes a través de las interacciones y prácticas de crianza, en este estudio planteamos la importancia de conocer y explicar en qué medida las prácticas parentales de padres y madres predicen la ideación suicida de los y las hijas adolescentes.

\section{MÉTODO}

\subsection{Diseño}

El diseño de investigación fue cuantitativo con alcance explicativo puesto que el objeto de estudio se planteó en términos de relación causal (Hernández-Sampieri, Fernández-Collado \& BaptistaLucio, 2010). Este proceso implicó realizar la descripción del fenómeno y el establecimiento de relaciones entre variables para realizar procedimientos orientados a establecer probables causas de la ideación suicida en adolescentes, las cuales se delimitaron desde las prácticas parentales.

\subsection{Participantes}

Para el desarrollo del presente estudio se seleccionó una muestra intencional por conveniencia, en la que no se determinó de inicio algún criterio de inclusión relacionado con las variables de estudio, en ella participaron 328 estudiantes adolescentes inscritos en escuelas públicas de la ciudad de Tunja, Colombia; de los cuales 175 (53,4 \%) eran mujeres y 153 (46,6 \%) hombres; el rango de edad fue de 15 a 18 años para hombres y mujeres; el promedio de edad para el grupo de hombres fue de 16 años (DE .8390); para el grupo de mujeres el promedio fue de 15,7 años (DE .7972). El nivel de escolaridad promedio fue de 10,5 años estudiados (DE .50031).

\subsection{Instrumentos}

Se utilizó la escala de prácticas parentales para adolescentes (PP-A) elaborada por Andrade y Betancourt (2008). Es un instrumento autoaplicable de 80 reactivos, 40 reactivos para el papá y 40 para la mamá, con cuatro opciones de respuesta: nunca, pocas veces, muchas veces y siempre. A continuación se describen las dimensiones que conforman cada escala. 


\section{Marly Johana Bahamón, Yolima Alarcón-Vásquez, Ana María Trejos Herrera, Lizeth Reyes Ruiz, J. Isaac Uribe Alvarado, Carolina García Galindo}

Tabla 1

Descripción dimensiones y características psicométricas del cuestionario PP-A

\begin{tabular}{|c|c|c|c|}
\hline Dimensiones & Definición de dimensión & Alfa & $\begin{array}{l}\text { No. } d e \\
\text { reactivos }\end{array}$ \\
\hline $\begin{array}{l}\text { Comunicación y control } \\
\text { conductual paterno }\end{array}$ & $\begin{array}{l}\text { Comunicación existente entre el padre y el } \\
\text { adolescente y Conocimiento que tienen el } \\
\text { padre de las actividades de sus hijos. }\end{array}$ & 0.97 & 16 \\
\hline Autonomía paterna & $\begin{array}{l}\text { El respeto que muestra el padre en las } \\
\text { decisiones que toman los hijos. }\end{array}$ & 0.94 & 8 \\
\hline Imposición paterna & $\begin{array}{l}\text { El grado en que el padre impone sus } \\
\text { creencias y conductas al hijo. }\end{array}$ & 0.90 & 8 \\
\hline Control psicológico paterno & $\begin{array}{l}\text { Inducción de culpa, devaluación y críticas } \\
\text { excesivas al adolescente. }\end{array}$ & 0.90 & 8 \\
\hline Comunicación materna & $\begin{array}{l}\text { Comunicación existente entre la madre y el } \\
\text { adolescente. }\end{array}$ & 0.92 & 10 \\
\hline Autonomía materna & $\begin{array}{l}\text { El respeto que muestra la madre en las } \\
\text { decisiones que toman los hijos. }\end{array}$ & 0.86 & 7 \\
\hline Imposición materna & $\begin{array}{l}\text { El grado en que la madre impone sus } \\
\text { creencias y conductas al hijo. }\end{array}$ & 0.81 & 8 \\
\hline Control psicológico materno & $\begin{array}{l}\text { Inducción de culpa, devaluación y críticas } \\
\text { excesivas al adolescente. }\end{array}$ & 0.80 & 8 \\
\hline Control conductual materno & $\begin{array}{l}\text { Conocimiento que tienen la madre y el hijo } \\
\text { sobre sus actividades. }\end{array}$ & 0.84 & 7 \\
\hline
\end{tabular}

Se utilizó también una escala para medir la Ideación Suicida, la cual está constituida por ocho ítems que refieren a ideas de quitarse la vida, por ejemplo: "he tenido ideas de quitarme la vida" y "pienso que mi vida ha sido un fracaso", tiene cinco opciones de respuesta ordinal que van de 1 = a nunca; hasta 5 = con mucha frecuencia (más de dos veces por semana). La escala tiene una fiabilidad de: $\alpha=.92$.

\subsection{Procedimiento}

Tanto la escala de parentales para adolescentes (PP-A) como la escala de ideación suicida fueron aplicadas de manera grupal. Previa autorización de las autoridades y directivas de las escuelas, se solicitó la participación voluntaria de los y las adolescentes, se les informó verbalmente de los propósitos del estudio y se hizo especial énfasis en el anonimato de cada participante y en la confidencialidad en el uso de la información. Posteriormente se le entregó a cada estudiante de los grupos el cuestionario que describe en la parte inicial los propósitos del mismo, el aviso de confidencialidad y anonimato y el consentimiento para contestarlo o no de manera voluntaria. Posteriormente se procedió a la aplicación solo con quienes desearon participar. Una vez que se aplicaron los cuestionarios a la muestra del estudio, se procedió a su captura y análisis utilizando para ello el programa SPSS, versión 15, de Windows. Los estadígrafos fueron el coeficiente de correlación de Pearson y el análisis de regresión lineal.

\subsection{Análisis de datos}

Se realizaron análisis descriptivos de las variables, correlación mediante uso del coeficiente de Pearson y análisis de regresión lineal. 


\section{Marly Johana Bahamón, Yolima Alarcón-Vásquez, Ana María Trejos Herrera, Lizeth Reyes Ruiz, J. Isaac Uribe Alvarado, Carolina García Galindo}

\section{RESULTADOS}

Primeramente, se describen algunas características de la estructura familiar de los y las adolescentes participantes, en el grupo de hombres, el 90,8\% vive con papá y mamá, el 5,2 \% solo con la mamá y el 3,9 \% solo con el papá. Para el grupo de mujeres, el 85,7 \% vive con ambos padres, el 11,4 \% vive solo con mamá y el 2,9 \% solo con papá. No se reportó si tienen hermanos o hermanas.

Con el fin de conocer la diferencia en los promedios de la ideación suicida en hombres y mujeres se realizó una prueba t de Student (ver Tabla 2).

Tabla 2

Comparación de promedios del cuestionario de ideación suicida por sexo.

\begin{tabular}{llllllll}
\hline Variable & Sexo & N & Media & D.E. & t & gl & p \\
\hline Ideación suicida & $H$ & 153 & 9.562 & 3.390 & & & \\
\hline
\end{tabular}

Con el propósito de determinar la relación entre las prácticas parentales con la ideación suicida de los participantes, se llevaron a cabo correlaciones de Pearson; estos análisis se hicieron separados para hombres como para mujeres adolescentes. Se reportan correlaciones entre prácticas parentales maternas e ideación suicida para ambos grupos (ver Tabla 3).

Tabla 3

Correlación entre ideación suicida y prácticas parentales maternas

\begin{tabular}{|c|c|c|c|c|c|c|}
\hline & 1. & 2. & 3. & 4. & 5. & 6. \\
\hline 1. Ideación suicida & --- &,- 149 &,$- 303^{* *}$ &,- 137 & ,302** &,- 070 \\
\hline 2. Comunicación & .074 & --- &, $686^{* *}$ &, $711^{* *}$ &,$- 217^{* *}$ &, $347 * *$ \\
\hline 3. Autonomía & -.106 & $.389^{* *}$ & --- &, $661^{* *}$ &,$- 337 * *$ &, $217^{* *}$ \\
\hline 4. Imposición & -.059 & $.396^{* *}$ & $.406^{* *}$ & --- &,$- 161^{*}$ & ,305** \\
\hline 5. Control psicológico & $.183^{*}$ & -.054 & -.146 & .164 & --- & 100 \\
\hline 6. Control conductual & -.078 & $.336^{* *}$ & $.326^{* *}$ & $.210^{*}$ & .128 & --- \\
\hline
\end{tabular}

Para el grupo de hombres es de destacarse la correlación positiva baja entre ideación suicida y control psicológico materno y para el grupo de mujeres la correlación negativa entre ideación suicida y autonomía materna así como una correlación positiva entre ideación suicida y control psicológico materno (ver Tabla 4).

Tabla 4.

Correlación entre ideación suicida y prácticas parentales paternas en hombres y mujeres.

\begin{tabular}{|c|c|c|c|c|c|}
\hline & 1. & 2. & 3. & 4. & 5. \\
\hline 1. Ideación suicida & 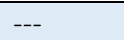 & $162^{*}$ &,- 133 &,$- 383^{* *}$ &,- 081 \\
\hline $\begin{array}{l}\text { 2. Comunicación y control } \\
\text { conductual }\end{array}$ & ,197* & --- &, $471 * *$ &, $340 * *$ &, $524 * *$ \\
\hline 3. Autonomía &,- 019 &, $583 * *$ & --- &, $618 * *$ &, $555^{* *}$ \\
\hline 4. Imposición &,$- 285^{* *}$ &, $355^{* *}$ &, $629 * *$ & -- &, $615^{* *}$ \\
\hline 5. Control psicológico & 103 &, $563 * *$ &, $681^{* *}$ &, $431^{* *}$ & --- \\
\hline
\end{tabular}




\section{Marly Johana Bahamón, Yolima Alarcón-Vásquez, Ana María Trejos Herrera, Lizeth Reyes Ruiz, J. Isaac Uribe Alvarado, Carolina García Galindo}

Respecto a las correlaciones entre prácticas parentales de los padres y la ideación suicida, para el grupo de hombres se reporta una correlación negativa significativa entre la ideación suicida y la imposición; para el grupo de mujeres se reporta esta misma correlación con diferente intensidad.

\subsection{Predictores de la ideación suicida}

Se realizaron análisis de regresión lineal con el método de pasos sucesivos para determinar cuáles de las dimensiones de las prácticas parentales predicen la ideación suicida tanto en hombres como en mujeres participantes en el estudio. Teniendo en cuenta que el valor más bajo en la escala es de 8 y el más alto es de 40 puntos, los valores de referencia oscilan entonces entre $8=$ nunca ha tenido ideas suicidas; de 9 a 16 puntos = casi nunca ha tenido ideas suicidas; de 17 a $24=$ algunas veces ha tenido ideas suicidas; de 25 a 32 con frecuencia he tenido ideas suicidas y de 33 a 40 puntos = con mucha frecuencia he tenido ideas suicidas.

Los resultados de estos análisis indican que, respecto a las prácticas parentales maternas, la dimensión control psicológico predice en un $20 \%$ la ideación suicida en el grupo de hombres; para el grupo de mujeres, esta misma dimensión predice en un 30 \% la ideación suicida (ver Tabla 4). El control psicológico materno predice entonces la ideación suicida tanto en hombres como en mujeres adolescentes.

Tabla 5.

Practicas parentales de mamá como predictoras ideación suicida en hombres y mujeres

\begin{tabular}{|c|c|c|c|c|c|c|}
\hline Sexo & Variables independientes predictoras & $\mathrm{R}^{2}$ & 回 & $F$ & $g l$ & $p$ \\
\hline Hombres & Control psicológico & $.201^{a}$ & .201 & 5.063 & 121 & .026 \\
\hline \multirow[t]{2}{*}{ Mujeres } & Control psicológico & $.302^{\mathrm{a}}$ & .302 & 14.358 & 144 & .000 \\
\hline & Control psicológico, Promoción de la autonomía. & $.359^{b}$ &,- 206 & 10.508 & 144 & .000 \\
\hline
\end{tabular}

Respecto a las prácticas parentales paternas (Tabla 6), en el grupo de hombres la dimensión imposición predice en un primer modelo un $28 \%$ la ideación suicida; en un segundo modelo la imposición sumada a la comunicación y control conductual paterno predicen en un 42 \% la ideación suicida. Estas mismas dimensiones (Imposición; Comunicación y control conductual) predicen la ideación suicida en el grupo de mujeres en un 47 \%. Lo anterior indica que mientras mayor sea la imposición así como el control conductual de papás es altamente predecible que se presenten ideas suicidas en los adolescentes de ambos sexos.

Tabla 6

Practicas parentales de papá como predictoras ideación suicida en hombres y mujeres

\begin{tabular}{ll|lllll}
\hline Sexo & Variables independientes predictoras & $\mathrm{R}^{2}$ & l & $F$ & $g /$ & $P$ \\
\hline Hombres & Imposición & $.283^{\mathrm{a}}$ & -.283 & 130 & 11.230 & $.001^{\mathrm{a}}$ \\
& Imposición & $.428^{\mathrm{b}}$ & -411 & 130 & 14.376 & $.000^{\mathrm{b}}$ \\
& Comunicación, & & .346 & & & \\
& Control conductual & & & & & \\
Mujeres & Imposición & $.367^{\mathrm{a}}$ & -.367 & 144 & 22.467 & $.000^{\mathrm{a}}$ \\
& Comunicación, & $.477^{\mathrm{b}}$ & -.473 & 145 & 21.089 & $.000^{\mathrm{b}}$ \\
& Control conductual & & .322 & & & \\
\hline
\end{tabular}




\section{Marly Johana Bahamón, Yolima Alarcón-Vásquez, Ana María Trejos Herrera, Lizeth Reyes Ruiz, J. Isaac Uribe Alvarado, Carolina García Galindo}

Una vez conocidas las dimensiones de las prácticas parentales de papás y mamás que predicen la ideación suicida en hijos adolescentes, y con el propósito de determinar de manera conjunta cuáles prácticas parentales predicen la ideación suicida, se realizó una regresión en la cual se plantearon las nueve prácticas parentales de forma conjunta. Se reporta que para los hombres, como para el grupo de mujeres, las dimensiones que predicen la ideación suicida son la imposición paterna, comunicación y control conductual paterno en $45 \%$ en hombres y $49 \%$ en mujeres; solo en el caso de las mujeres y sumado a las dimensiones ya mencionadas el control psicológico materno se suma a las anteriores con 2,5\% dando un porcentaje predictivo total de $52 \%$ para el tercer modelo en el grupo de mujeres (Tabla 7). Lo anterior indica que las dimensiones de imposición, y comunicación y control conductual de los papás tienen mayor poder predictivo de la ideación suicida en hombres y mujeres adolescentes que las prácticas parentales maternas.

Tabla 7

Practicas parentales de mamá y papá predictoras ideación suicida en hombres y mujeres

\begin{tabular}{lllllll}
\hline Sexo & Variables independientes predictoras & $\mathrm{R}^{2}$ & l & $F$ & $g l$ & $p$ \\
\hline Hombres & Imposición paterna & $.278^{\mathrm{a}}$ & -.278 & 107 & 8.846 & $.004^{\mathrm{a}}$ \\
& Comunicación, & $.455^{\mathrm{b}}$ & -.442 & 107 & 13.676 & $.000^{\mathrm{b}}$ \\
& Control conductual paterna & & .396 & & & \\
Mujeres & Imposición paterna & $.389^{\mathrm{a}}$ & -.389 & 127 & 22.518 & $.000^{\mathrm{a}}$ \\
\hline & Comunicación, & $.495^{\mathrm{b}}$ & -.507 & 127 & 20.287 & $.000^{\mathrm{b}}$ \\
& Control conductual paterno & & .328 & & & \\
& Comunicación, & $.520^{\mathrm{c}}$ & -.455 & 127 & 15.344 & $.000^{\mathrm{c}}$ \\
& Control conductual paterno & & .275 & & & \\
\hline
\end{tabular}

\section{DISCUSIÓN}

La ideación suicida se constituye en una de las fases iniciales del proceso suicida, en tanto, invade el pensamiento del sujeto con ideas referentes a la muerte y a la falta de sentido para vivir. No obstante, involucra fenómenos complejos que hacen más vulnerables a las personas para llevar a cabo el intento de quitarse la vida. La ideación suicida se encuentra presente de manera ineludible en quienes han ejecutado el hecho de quitarse la vida y por tanto se convierte en una etapa que permite la detección temprana de factores de riesgo que pueden intervenirse oportunamente para evitar la transición al intento suicida o al suicidio consumado.

Los estudios relacionados con el tema reportan que la familia es fundamental como elemento moderador de la ideación e intento suicida en los adolescentes. Así, la percepción negativa sobre esta, el rechazo por parte de los padres y las discusiones se asocian con la salud mental del adolescente, específicamente con la presencia de depresión, ideación e intento suicida (Monge, Cubillas, Román \& Abril, 2007). Al respecto es importante referir que el estudio realizado se encuentra en la misma línea puesto que se detectaron relaciones significativas entre algunas prácticas ejercidas por los padres y la presencia de ideación suicida.

En este sentido, los resultados de la investigación mostraron una correlación significativa entre la ideación suicida y el control psicológico, la autonomía y la imposición de los padres. Resultados que ponen en evidencia como algunos patrones de comportamiento regulares en los padres al ejercer 


\section{Marly Johana Bahamón, Yolima Alarcón-Vásquez, Ana María Trejos Herrera, Lizeth Reyes Ruiz, J. Isaac Uribe Alvarado, Carolina García Galindo}

su rol dentro del proceso de formación de los adolescentes se encuentran involucrados como un posible factor de riesgo para disponer a los jóvenes hacia el suicidio. Así, emerge el papel fundante de la familia en el desarrollo del joven y su función socializadora que hace más vulnerable al sujeto hacia determinadas formas de percibirse a sí mismo y al mundo. Interpretaciones que pueden impulsar visiones positivas o negativas sobre las capacidades del joven para afrontar las demandas del medio, sentidos y expectativas hacia el presente y el futuro (Kwok \& Shek, 2009).

Así, al analizar las prácticas parentales predictoras de la ideación suicida en los adolescentes participantes los resultados mostraron que la imposición autoritaria de los padres sobre sus hijos, la comunicación y el control conductual aumentaban en un alto porcentaje la posibilidad de ideación suicida tanto en hombres como mujeres.

Lo anterior, sugiere que los adolescentes que han sido expuestos a la imposición de normas y reglas sin posibilidades de participación en ambientes caracterizados por el autoritarismo y a un excesivo control tienden a reducir el valor que le atribuyen a sus vidas. Estos resultados coinciden con lo encontrado por algunos estudios (Shur-Fen et al., 2008; Kim \& Kim, 2008), que indican que la sobreprotección y el control autoritario se relacionan significativamente con el riesgo suicida.

Estos datos traen a discusión el papel que los padres están ejerciendo sobre sus hijos y la intencionalidad de sus comportamientos, pues lo más probable es que es tos obedezcan a un interés por brindar oportunidades de desarrollo logrando un efecto contrario. En este orden de ideas, vale la pena explorar los miedos y atribuciones que los padres realizan sobre la educación de sus hijos y sus capacidades para enfrentar los múltiples retos que la vida les propone, así también emergen otros elementos que valdría la pena explorar en futuras investigaciones y que hacen referencia a los temores presentes en los padres y su entrenamiento para asumir a sus hijos como seres autónomos que requieren de orientación adecuada para aprender a tomar decisiones democráticamente sin llegar a límites que coincidan con la omisión y la permisividad.

Otro aspecto relevante mencionado en los resultados del estudio fue la comunicación debido a que esta se presenta como una variable que predice fuertemente la ideación suicida en los participantes, resultados que coinciden parcialmente con lo encontrado por Lai y Shek (2010), quienes realizaron un estudio sobre la ideación suicida y el funcionamiento familiar en general encontrando que solamente se evidenciaba una fuerte correlación entre la comunicación y la ideación suicida en los jóvenes cuando se trataba de la madre pero no del padre. Dichos hallazgos requieren mayor análisis, debido a que los resultados de este estudio sugieren que no solo la comunicación con la madre es fundamental sino además, con el padre. Así, es posible afirmar que el papel del padre en los procesos de prevención puede ser más activo que en otros países, como es el caso de Oriente. De esta forma, los datos exponen la necesidad de involucrar al padre en los procesos de formación que se brindan en diferentes contextos y que contribuyen en la prevención del suicidio.

Finalmente, otro elemento que aparece en los resultados es el control psicológico, el cual, según los resultados de esta investigación ejerce mayor riesgo sobre las mujeres que sobre los hombres y es 


\section{Marly Johana Bahamón, Yolima Alarcón-Vásquez, Ana María Trejos Herrera, Lizeth Reyes Ruiz, J. Isaac Uribe Alvarado, Carolina García Galindo}

frecuentemente utilizado por la madre. Este tipo de práctica implica un proceso de control pasivo que se constituye en comportamientos coercitivos y hostiles manifiestos en inducción de culpa, invalidación de sentimientos y sobreprotección que si se presentan de manera consistente en los padres se asocia a problemas de comportamiento y emocionales en los hijos (Betancourt \& Andrade, 2011; Lai \& McBride, 2001). Este tipo de control es diferente al control conductual, el cual involucra mayor atención de los padres hacia las actividades de sus hijos y su respectiva supervisión.

Lo anterior, evidencia la importancia de proponer a los padres mecanismos de formación ligados a la presencia y acompañamiento continuo en las actividades que desarrollan sus hijos permitiéndoles notar su papel orientador sin obstaculizar el desarrollo de su autonomía e independencia, ni utilizar mecanismos violentos o manipuladores. Así, el establecimiento de reglas, la exposición a normas claras y la supervisión continua reduciría la necesidad de usar chantajes emocionales para controlar el comportamiento de los hijos y de paso facilitar la presencia de problemas emocionales que pueden derivar en ideación o conductas suicidas.

Es importante considerar que el alcance de las conclusiones se limita a la muestra misma, y se recomienda que en estudios similares se considere dentro de lo posible utilizar muestras aleatorias para mayor generalización y aplicación de los resultados; así mismo, reconocemos que el autoreporte de los adolescentes sobre las prácticas parentales puede verse como limitación en este estudio, por lo que recomendamos desarrollar estrategias de obtención de información de padres y madres respecto a la crianza de sus hijos e hijas adolescentes, retomando siempre una perspectiva sociocultural y de género.

Nota de Autores

Este artículo se deriva del proyecto de investigación titulado "Conductas y prácticas de riesgo en adolescentes

\section{REFERENCIAS}

Álvarez, M., Ramírez, B., Silva, A., Coffin, N. \& Jiménez, M. (2009). La relación entre depresión y conflictos familiares en adolescentes. International Journal of Psychological Therapy, 09(2), 205-216. Disponible en http://www.ijpsy.com/volumen9/num2/232/la-relacin-entre-depresin-y-conflictos-ES.pdf

Andrade, P. \& Betancourt, O. (2008). Prácticas parentales: Una medición integral. La Psicología Social en México, XII, 561-565.

AMEPSO. Atay, M., Eren, I., \& GündoĞar, D. (2012). The prevalence of death Ideation and attempted suicide and the associated risk factors in Isparta, Turkey. Turkish Journal of Psychiatry, 23(2), 89-98. Dsiponible en https://www.ncbi.nlm.nih.gov/pubmed/22648871 


\section{Marly Johana Bahamón, Yolima Alarcón-Vásquez, Ana María Trejos Herrera, Lizeth Reyes Ruiz, J. Isaac Uribe Alvarado, Carolina García Galindo}

Betancourt, D., \& Andrade, P. (2011). Control parental y problemas emocionales y de conducta en adolescentes. Revista Colombiana de Psicología, 20(1), 27-41. Dsiponible en https://revistas.unal.edu.co/index.php/psicologia/article/view/17448/28672

Casey, P., Dunn, G., \& Kelly, B. (2006). Factors associated with suicidal ideation in the general population: five- center analysis from the ODIN study. The British Journal of Psychiatry, (189), 410-15. https://doi.org/10.1192/bjp.bp.105.017368

Casullo, M., Bonaldi, D., \& Fernández, M. (2000). Comportamiento suicida en la adolescencia: morir antes de la muerte. Editorial Buenos Aires.

Cavanagh, J., Carson, A. \& Sharpe, M. (2003). Psychological autopsy studies of suicide: a systematic review. Psychological Medicine, 33(3), 395-405. https://doi.org/10.1017/S003329170200694360

Caycedo, A., Arenas, M., Benítez, M., Cavanzo, P., Leal, G. \& Rossini, Y. (2010). Características psicosociales y familiares relacionadas con intento de suicidio en una población adolescente en Bogotá-2009. Revista Persona y Bioética, 14(2), 205-213. DOI: 10.5294/pebi.2010.14.2.8

Cortés, A., Aguilar, J., Suárez, R., Rodríguez, E. \& Salvador, J. (2011). Factores de riesgo asociados con el intento suicida y criterios sobre lo ocurrido en adolescentes. Revista Cubana de Medicina General Integral, 27(1), 33-41. Dsiponible en http://www.bvs.sld.cu/revistas/mgi/vol_27_1_11/mgi04111.htm

Cummings, M. \& Davies, T. (2002). Effects of marital conflicts on children: recent advances and emerging themes in process-oriented research. Journal of Child Psychology and Psychiatry, 43, 31-63. DOI: 10.1111/14697610.00003.

Ferran, J., Canals, Mạ. Gras, C. \& Domènech-Llaberia, E. (2002). Psychological and Family Factors Associated with Suicidal Ideation in Pre-Adolescents. The Spanish Journal of Psychology, 5(1), 20-28. https://doi.org/10.1017/S1138741600005795

Florenzano, R., Valdés, M., Cáceres, E., Santander, S., Aspillaga, C. \& Musalem, C. (2011). Relación entre ideación suicida y estilos parentales en un grupo de adolescentes chilenos. Revista Médica de Chile, 139(12), 1529-1533. Dsiponible en http://www.revistamedicadechile.cl/ojs/index.php/rmedica/article/view/1451

Hernández-Sampieri, R., Fernández-Collado, C. \& Baptista-Lucio, P. (2010). Metodología de la investigación. México: McGraw-Hill.

Herrera, P. \& Avilés, K. (2000). Factores familiares de riesgo en el intento suicida. Revista Cubana de Medicina General Integral, 16(02), 134-137. Dsiponible en http://repositorio.ucp.edu.co:8080/jspui/bitstream/10785/3297/1/ARTICULO\%20TERMINADO.pdf

Instituto de Medicina Legal y Ciencias Forenses (2015). Datos oficiales sobre la violencia en Colombia, 2015. Revista Forensis. Disponible en: http://www.medicinalegal.gov.co/documents/88730/3418907/7.+SUICIDIOS.pdf/01c41af2-27cf4932-ae14234d1eeaf425

Kim, H., \& Kim, E. (2008). Risk Factors for Suicide Attempts among Korean Adolescents. Child Psychiatry \& Human Development, 39(3), 221-235. DOI: 10.1007/s10578-007-0083-4

Kwok, S. \& Shek, D. (2009). Social problem solving, family functioning, and suicidal ideation among chinese adolescents in Hong Kong. Adolescence, 44(174), 391-406. Dsiponible en https://www.ncbi.nlm.nih.gov/pubmed/19764274 


\section{Marly Johana Bahamón, Yolima Alarcón-Vásquez, Ana María Trejos Herrera, Lizeth Reyes Ruiz, J. Isaac Uribe Alvarado, Carolina García Galindo}

. Lai, K. \& McBride, K. (2001). Suicidal ideation, parenting style, and family climate among Hong Kong adolescents. International Journal of Psychology, 36(2), 81-87. https://doi.org/10.1080/00207590042000065

Lai, S., Kwok, S. \& Shek, D. (2008). Hopelessness, Family Functioning and Suicidal Ideation Among Chinese Adolescents in Hong Kong. The Open Family Studies Journal, (1), 49-55. DOI: 10.2174/1874922400801010049

Lai, S. \& Shek, D. (2010). Hopelessness, Parent-Adolescent Communication, and Suicidal Ideation among Chinese Adolescents in Hong Kong. Suicide \& Life. Threatening Behavior, 40(3), 224-33. DOI: 10.1521/suli.2010.40.3.224

Monge, J., Cubillas., M., Román, R. \& Abril, E. (2007). Intentos de suicidio en estudiantes de educación media superior. Y su relación con la familia. Revista Psicología y salud, 17(1), 45-51. Dsiponible en http://revistas.uv.mx/index.php/psicysalud/article/view/738

Palacios, R. \& Andrade, P. (2008). Influencia de las prácticas parentales en las conductas problema en adolescentes. Revista Investigación Universitaria Multidisciplinaria, 7(7), 7-18. Dsiponible en http://biblioteca.universia.net/html_bura/ficha/params/title/influencia-practicas-parentales-conductasproblema-adolescentes/id/44833363.html

Pérez, A., Uribe, J., Vianchá, M., Bahamón, M., Verdugo, J. \& Ochoa, S. (2013). Estilos parentales como predictores de ideación suicida en estudiantes adolescentes. Psicología desde el Caribe, 30(3), 551- 568. Dsiponible en http://www.redalyc.org/articulo.oa?id=21329176006

Pérez-Olmos, I., Ibáñez-Pinilla, M., Reyes-Figueroa, J., Atuesta-Fajardo, J. \& Suárez-Díaz, M. (2008). Factores asociados al intento suicida e ideación suicida persistente en un centro de atención primaria. Bogotá, 2004-2006. Revista de Salud Pública, 10(3), 374-385. Dsiponible en https://www.scielosp.org/pdf/rsap/2008.v10n3/374-385

Reyes, W. \& Torres, N. (2001). Intento suicida y funcionamiento familiar. Revista Cubana de Medicina General Integral, 17(5), 452-460. Dsiponible en http://scielo.sld.cu/scielo.php?script=sci_arttext\&pid=S086421252001000500008

Rivera, M. \& Andrade, P. (2006). Recursos individuales y familiares que protegen al adolescente del intento suicida. Revista Intercontinental de Psicología y Educación, 8(2), 23-40. Dsiponible en http://journaldatabase.info/articles/recursos_individuales_y_familiares_que_protegen.html

Salvo, L. \& Melipillán, R. (2008). Predictores de suicidalidad en adolescentes. Revista Chilena de NeuroPsiquiatría, 46(2), 115-123. Dsiponible en http://www.revistachilenadeepilepsia.cl/wpcontent/uploads/2014/08/201302_suicidalidad_adolescentes.pdf

Sánchez-Sosa, J., Villarreal-González, M., Musitu, G., \& Martínez, B. (2010). Ideación suicida en adolescentes: Un análisis psicosocial. Revista Intervención Psicosocial, 19(3), 279-287. https://doi.org/10.5093/in2010v19n3a8

Santos, B., Ferrera, A., González, P., Viñas, G. \& Mauriset, R. (1997). Tentativa de suicidio y Apgar familiar modificado. Revista Cubana de Medicina General Integral, 13(4). Dsiponible en http://scielo.sld.cu/scielo.php?script=sci_arttext\&pid=S0864-21251997000400002

Sarmiento, C. \& Aguilar, J. (2011). Predictores familiares y personales de la ideación suicida en adolescentes. Revista $\begin{array}{lllll}\text { Psicología } \quad \text { y } & \text { 21(1), } & \text { 25-30. Dsiponible en }\end{array}$ http://revistas.uv.mx/index.php/psicysalud/article/view/583/1006 


\section{Marly Johana Bahamón, Yolima Alarcón-Vásquez, Ana María Trejos Herrera, Lizeth Reyes Ruiz, J. Isaac Uribe Alvarado, Carolina García Galindo}

Shur-Fen, S., Ying-Yeh, Ch., Fang-Ju, T., Ming-Been, L., Yen-Nan, Ch., Wei-Tsuen, S. \& Hai-Gwo, H. (2008). Risk factors for suicida in Taiwanese college students. Journal of American College Health, 57(2), $135-142$. https://doi.org/10.3200/JACH.57.2.135-142

Sook, H., Young, H. \& Schepp, K. (2005). Predictors of Suicidal Ideation for Adolescents by Gender. Journal of Korean Academy of Nursing, 35(8), 1433-1442. https://doi.org/10.4040/jkan.2005.35.8.1443

Suominen, K., Isometsä, E., Suokas, J., Haukka, J., Achte, K. \& Lönnqvist, J. (2004). Completed suicide after a suicide attempt: a 37- year follow up study. The American Journal of Psychiatry, 161(3), 562-3.DOI: 10.1176/appi.ajp.161.3.562

Toro, D., Paniagua, R., González, C. \& Montoya, B. (2009). Caracterización de adolescentes escolarizados con riesgo de suicidio, Medellín, 2006. Revista Fac. Nac. Salud Pública, 27(3), 302-308. Dsiponible en http://aprendeenlinea.udea.edu.co/revistas/index.php/fnsp/article/view/1446

Está obra está bajo: Creative commons attribution 4.0 international license. El beneficiario de la licencia tiene el derecho de copiar, distribuir, exhibir y representar la obra y hacer obras derivadas siempre y cuando reconozca y cite la obra de la forma especificada por el autor o el licenciante

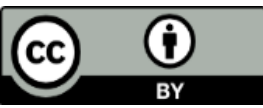

